\title{
Alcohol and trauma: the influence of blood alcohol levels on the severity of injuries and outcome of trauma patients - a retrospective analysis of 6268 patients of the TraumaRegister DGU
}

Thomas Brockamp ${ }^{1,4^{*}} \mathbb{D}$, Andreas Böhmer², Rolf Lefering ${ }^{3}$, Bertil Bouillon ${ }^{1}$, Arasch Wafaisade ${ }^{1}$, Manuel Mutschler ${ }^{1}$, Paola Kappel ${ }^{1}$, Matthias Fröhlich ${ }^{1}$ and Working Group of Injury Prevention of the German Trauma Society (DGU) ${ }^{3}$

\begin{abstract}
Background: Blood alcohol level (BAL) has previously been considered as a factor influencing the outcome of injured patients. Despite the well-known positive correlation between alcohol-influenced traffic participation and the risk of accidents, there is still no clear evidence of a positive correlation between blood alcohol levels and severity of injury. The aim of the study was to analyze data of the TraumaRegister DGU (TR-DGU), to find out whether the blood alcohol level has an influence on the type and severity of injuries as well as on the outcome of multiple-trauma patients.

Methods: Datasets from 11,842 trauma patients of the TR-DGU from the years 2015 and 2016 were analyzed retrospectively and 6268 patients with a full dataset and an AIS $\geq 3$ could be used for evaluation. Two groups were formed for data analysis. A control group with a BAL $=0 \%$ (BAL negative) was compared to an alcohol group with a BAL of $\geq 0.3 \%$ to $<4.0 \%$ (BAL positive). Patients with a BAL $>0 \%$ and $<0.3 \%$ were excluded. They were compared with regard to various preclinical, clinical and physiological parameters. Additionally, a subgroup analysis with a focus on patients with a traumatic brain injury (TBI) was performed.

A total of 5271 cases were assigned to the control group and 832 cases to the BAL positive group. 70.3\% (3704) of the patients in the control group were male. The collective of the control group was on average 5.7 years older than the patients in the BAL positive group $(p<.001)$. The control group showed a mean ISS of 20.3 and the alcohol group of $18.9(p=.007)$. In terms of the injury severity of head, the BAL positive group was significantly higher on average than the control group $(p<0.001)$, whereas the control group showed a higher AIS to thorax and extremities $(p<0.001)$. The mean Glasgow Coma Scale (GCS) was 10.8 in the BAL positive group and 12.0 in
\end{abstract}

\footnotetext{
* Correspondence: tbrockamp@gmail.com

'Department of Trauma and Orthopaedic Surgery, University of Witten/ Herdecke, Cologne-Merheim-Medical-Center, Ostmerheimer Street 200, 51109 Cologne, Germany

${ }^{4}$ Working Group of Injury Prevention of the German Trauma Society, The German Trauma Society (DGU), Straße des 17. Juni 106-108, 10623 Berlin, Germany

Full list of author information is available at the end of the article
}

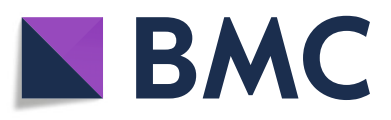

(c) The Author(s). 2021 Open Access This article is licensed under a Creative Commons Attribution 4.0 International License, which permits use, sharing, adaptation, distribution and reproduction in any medium or format, as long as you give appropriate credit to the original author(s) and the source, provide a link to the Creative Commons licence, and indicate if changes were made. The images or other third party material in this article are included in the article's Creative Commons licence, unless indicated otherwise in a credit line to the material. If material is not included in the article's Creative Commons licence and your intended use is not permitted by statutory regulation or exceeds the permitted use, you will need to obtain permission directly from the copyright holder. To view a copy of this licence, visit http://creativecommons.org/licenses/by/4.0/ The Creative Commons Public Domain Dedication waiver (http://creativecommons.org/publicdomain/zero/1.0/) applies to the data made available in this article, unless otherwise stated in a credit line to the data. 


\begin{abstract}
the control group $(p<0.001)$. Physiological parameters such as base excess (BE) and International Normalized Ratio (INR) showed reduced values for the BAL positive group. However, neither the 24-h mortality nor the overall mortality showed a significant difference in either group ( $p=0.19, p=0.14)$. In a subgroup analysis, we found that patients with a relevant head injury (AIS: Abbreviated Injury Scale head $\geq 3$ ) and positive BAL displayed a higher survival rate compared to patients in the control group with isolated TBI $(p<0.001)$.

Conclusions: This retrospective study analyzed the influence of the blood alcohol level in severely injured patients in a large national dataset. BAL positive patients showed worse results with regard to head injuries, the GCS and to some other physiological parameters. Finally, neither the 24-h mortality nor the overall mortality showed a significant difference in either group. Only in a subgroup analysis the mortality rate in BAL negative patients with TBI was significantly higher than the mortality rate of BAL positive patients with TBI. This mechanism is not yet fully understood and is discussed controversially in the literature.
\end{abstract}

Keywords: Blood alcohol, Traumatic brain injury, Outcome, Youth

\section{Background}

Every year, 1.2 million people worldwide die in road accidents [1]. Across the EU, more than 30,000 people die each year in road accidents. Millions more suffer injuries and permanent disabilities. Alcohol and drug abuse are often the causes of serious road accidents [2]. In Germany, a total of 2,5 million accidents were recorded by the police in 2018, including 37,450 accidents in which at least one person was under the influence of intoxicating substances. Alcohol influence was one of the causes of accidents in 2017 for $4.1 \%$ of all accidents in which persons have been involved. Overall, $7.4 \%$ of all fatally injured road users in Germany died as a result of an accident due to alcohol abuse. The number of accidents under the influence of other intoxicating substances increased sharply between 1991 and 2017 and almost quadrupled, from 434 to 1679 accidents [3].

Despite the well-known positive correlation between alcohol-influenced traffic participation and the risk of accidents, there is still no clear evidence of a positive correlation between blood alcohol levels (BAL) and severity of injury. Moreover, it is not clear what precise influence the BAL has on the outcome (mortality; hospital stay, etc.) of a trauma patient injured in a traffic accident. Several studies have shown that patients with acute alcohol intoxication have a shorter hospital stay than patients without alcohol exposure [4-6]. However, other authors could not prove a link between alcohol misuse and the severity of injury or outcome $[7,8]$ or show that alcohol consumption increases the severity of injury [9]. The reasons for these different results can certainly also be found in the retrospective study designs and applied methodologies. Nevertheless, it is important to further analyze the effects of alcohol and drugs on the human body in the context of a seriously injured person in order to understand existing mechanisms of action. We think that our work can help to underline the important relation between the influence of alcohol on the body in multiple trauma patients. It is not yet sufficiently understood how exactly alcohol changes the physiology of different systems in the human body (e.g. brain; blood; coagulopathy; blood pressure). To our knowledge, the literature does not give an adequate answer and in vitro studies on multiple trauma patients with a measurable blood alcohol level are rare.

The aim of the present study was to check whether the blood alcohol level (measured in permille) has an influence on the nature and severity of injuries as well as on the outcome of trauma patients. A retrospective analysis using data of the TR-DGU was performed.

\section{Methods}

In this retrospective observational study, data of 11,842 trauma patients from the TraumaRegister $\mathrm{DGU}^{\circ}$, collected between 2015 and 2016, were analyzed. The alcohol parameter was included in the registry in 2015. 6103 data sets were used for data analysis. Only primary admitted patients with an AIS $\geq 3$ for whom a measured blood alcohol level was documented, were included. Patients transferred out early $(<48 \mathrm{~h})$ to another hospital (missing outcome) or patients who were secondary admitted (missing initial BAL) were not included.

Two groups were formed for data analysis. A control group with a BAL $=0 \%$ (BAL negative) and an alcohol group with a BAL of $\geq 0.3 \%$ to $<4.0 \%$ (BAL positive). Values of $4 \%$ or higher were considered unreliable and therefore excluded. The lower limit value $(0.3 \%$ ) was chosen because there are already measurable effects on the body. Patients with a BAL $>0 \%$ and $<0.3 \%$ were excluded. Additionally, a subgroup analysis was done to investigate the effect of alcohol in a dataset of patients with a severe head injury (AIS head $\geq 3$ ).

Preclinical, clinical and physiological parameters were compared, as were different outcome parameters between the two groups. Differences between the groups were analyzed at a significance level $p<0.05$ using the 
Mann-Whitney-U and Pearson's Chi-Squared test when appropriate. The data is presented as mean with standard deviation for continuous variables and as a percentage for categorical variables. The statistical analysis was performed using standard statistics software (SPSS version 24.0, IBM, Armonk, NY, USA).

\section{TraumaRegister DGU ${ }^{\circledast}$}

The TraumaRegister DGU of the German Society of Trauma Surgery (DGU) was founded in 1993. The aim of this multicentre database is to provide pseudonymised and standardised documentation of seriously injured persons.

The data are collected prospectively in four successive phases: A) preclinical phase, B) shock room and subsequent surgical phase, C) intensive care unit and D) discharge. The documentation contains detailed information on demography, injury patterns, comorbidities, preclinical and clinical management, intensive medical history, important laboratory findings including transfusion data, and outcome. The inclusion criterion is admission to the hospital via the shock room followed by intensive or intermediate care unit monitoring, or arrival in the clinic with vital signs and death before admission to the intensive care unit.

The infrastructure for documentation, data management and data analysis is provided by the AUC - Akademie der Unfallchirurgie $\mathrm{GmbH}$, which is affiliated with the DGU.

The scientific leadership is provided by the Committee on Emergency Medicine, Intensive.

Care and Trauma Management (Sektion NIS) of the German Trauma Society. Through a web-based application, the participating clinics enter their data pseudonymously into a central database. Scientific evaluations are approved according to a review procedure of the NIS section. The participating clinics are primarily located in Germany (90\%) but an increasing number of clinics from other countries also contribute data (currently from
Austria, Belgium, China, Finland, Luxembourg, Slovenia, Switzerland, the Netherlands and the United Arab Emirates). Currently, about 33,000 cases of more than 650 clinics are included in the database each year.

This study complies with the publication guidelines of the TraumaRegister DGU ${ }^{\circ}$ and is registered as TR-DGU Project ID 2017-020 N.

\section{Results}

A total of 5271 cases were assigned to the control group and 832 cases to the BAL positive group. Basis study characteristics are provided in Table 1 . It shows that $70 \%$ of the patients in the control group were male. The collective of the control group was on average 6 years older than the patients in the BAL positive group $(p<.001)$. The evaluation of the physiological parameters showed a higher blood pressure level in the control group (133 versus $125 \mathrm{mmHg}$ ) and a low hemoglobin value (12.9 versus 13.4). Base excess showed significantly lower levels in the BAL positive group. With regard to the ISS, the control group shows an average value of 20.3 and the alcohol group of $18.9(p=.006)$. In addition, the average GCS was 10.8 in the BAL positive group and 12.1 in the control group $(\mathrm{p}<.001)$.

Regarding the injury severity in different body regions, we found significantly higher values regarding AIS head of BAL positive patients $(p<0.001)$ whereas AIS thorax and AIS extremities were significantly lower in the BAL positive group compared to the control group $(\mathrm{p}<$ 0.001 ). No significant differences could be measured for AIS abdomen (Table 2).

Table 3 gives an overview of the type of accident depending on BAL. A significantly increased proportion of BAL positive patients suffered from low falls, while mechanisms with increased force, such as car or motorcycle accidents, were less common in this group $(p<0.001)$.

Neither the 24-h mortality nor the overall mortality showed a significant difference in either group $(p=0.19$,

Table 1 Patient characteristic

\begin{tabular}{lllll}
\hline & & BAL negative & BAL positive & \multicolumn{1}{c}{-value } \\
\hline Male & $\mathrm{n}(\%)$ & $3704(70.3)$ & $699(84.0)$ & $<.001$ \\
Age (years) & $($ Mean, SD) & $51 \pm 22$ & $45 \pm 18$ & $<.001$ \\
GCS & $($ Mean, SD) & $12.0 \pm 4.1$ & $10.8 \pm 4.5$ & $<.001$ \\
systBP on admission & (Mean, SD) & $133 \pm 32$ & $125 \pm 29$ & $<.001$ \\
Hemoglobin (g/dl) & (Mean, SD) & $12.9 \pm 2.2$ & $13.4 \pm 2.1$ & $<.001$ \\
INR & (Mean, SD) & $1.20 \pm 0.54$ & $1.13 \pm 0.49$ & $<.001$ \\
BE (mmol/l) & (Mean, SD) & $-1.8 \pm 4.4$ & $-3.9 \pm 4.5$ & \\
ISS & (Mean, SD) & $20.3 \pm 12.6$ & $18.9 \pm 11.6$ & .001 \\
NISS & (Mean, SD) & $25.3 \pm 15.3$ & $24.9 \pm 15.9$ & .27 \\
\hline BAL, Bl
\end{tabular}

BAL, Blood Alcohol Levels; systBP, Systolic Blood Pressure; GCS, Glasgow Coma Scale; INR, International Normalized Ratio; BE, Base Excess; ISS, Injury Severity Score; NISS, New Injury Severity Score; SD Standard Deviation 
Table 2 AIS depending on BA

\begin{tabular}{lllll}
\hline Relevant injury $(\mathbf{A I S} \geq \mathbf{3})$ of & & BAL negative & BAL positive & p-value \\
\hline Head & $\mathrm{n}(\%)$ & $2092(39.7 \%)$ & $405(48.7 \%)$ & $267(32.1 \%)$ \\
Thorax & $\mathrm{n}(\%)$ & $2195(41.6 \%)$ & $77(9.3 \%)$ & $<.001$ \\
Abdomen & $\mathrm{n}(\%)$ & $570(10.8 \%)$ & $143(17.2 \%)$ & .18 \\
Extremities / pelvis & $\mathrm{n}(\%)$ & $1378(26.1 \%)$ & $<.001$ \\
\hline
\end{tabular}

BAL, Blood Alcohol Levels; AIS, Abbreviated Injury Scale

$p=0.14)$. No relevant differences were found for ventilation days and length of stay on intensive care unit (ICU) or length of stay in hospital (Table 4).

We further analyzed the influence of alcohol on trauma patients with a combination of a severe TBI (AIS head $\geq 3$ ) and a positive BAL. This subgroup analysis showed that the mortality rate in patients with a positive BAL and a severe TBI was significantly lower (16.8\%) compared to patients of the BAL negative group (24.3\%) $(p=.001)$ although the BAL positive group showed more isolated traumatic brain injuries (43\% versus $31 \%$, $p<.001)$. The ISS showed higher values in the BAL negative group (27 vs. $24, p<0.001$ ).

The Revised Injury Severity Classification (RISC II) Score was additionally used to compare the observed with the predicted mortality. However, predicted mortality differed by less than $2 \%$ from observed mortality in both groups. In the subgroup TBI observed mortality was about $2 \%$ better than predicted (BAL positive), but the $95 \%$ CI was too broad to prove this effect.

Figure 1 shows the relation between age and BAL positive and BAL negative patients. Younger patients $(<$ 15 years) as well as older patients ( $>60$ years) were more frequently found in the control group, whereas patients about 25 and 55 years show an increased proportion in the BAL positive group.

\section{Discussion}

The influence of blood alcohol on the human body has been described many times [10-12]. In addition to the negative effects on, among others, liver function or the

Table 3 Mechanism of Injury according to BAL

\begin{tabular}{lllll}
\hline & & BAL negative & BAL positive & $\boldsymbol{p}$-value \\
\hline Car & $\mathrm{n}(\%)$ & $1256(23 \%)$ & $122(17 \%)$ & $>0.05$ \\
Motorbike & $\mathrm{n}(\%)$ & $769(14 \%)$ & $53(6 \%)$ & $>0.05$ \\
Bicycle & $\mathrm{n}(\%)$ & $519(9 \%)$ & $70(8 \%)$ & $>0.05$ \\
Pedestrian & $\mathrm{n}(\%)$ & $366(7 \%)$ & $76(9 \%)$ & $>0.05$ \\
Fall from $>3 \mathrm{~m}$ & $\mathrm{n}(\%)$ & $913(17 \%)$ & $126(15 \%)$ & $>0.05$ \\
Fall from <3 m & $\mathrm{n}(\%)$ & $963(18 \%)$ & $262(30 \%)$ & $<0.001$ \\
Blunt trauma & $\mathrm{n}(\%)$ & $5050(97 \%)$ & $781(93 \%)$ & $<0.001$ \\
Penetrating trauma & $\mathrm{n}(\%)$ & $107(2 \%)$ & $44(5 \%)$ & $>0.05$ \\
\hline
\end{tabular}

BAL, Blood Alcohol Levels central nervous system caused by ethanol, there are studies that describe a protective effect on some aspects, such as the cardiovascular system [13]. Road accidents often occur under the influence of alcohol and drugs. The risk of a road accident increases with increasing inability to drive, caused by alcohol and drugs $[14,15]$. However, it is not fully understood what effect a positive BAL has on the physiological parameters and outcome of a seriously injured patient. For example, some authors describe that they measured a lower GCS and lower blood pressure parameters in patients with positive BAL compared to BAL negative patients, and finally observed an increased mortality rate for BAL positive patients [9]. A positive BAL can also affect the occurrence of a traumatic brain injury (TBI). In animal models, alcohol has shown both adverse and neuroprotective effects, while clinical studies analyzing acute and long-term neurological and behavioral effects of intoxication show no consistent results $[4,16,17]$. Our study results confirm some of the hypotheses described. The majority of our study collective consists of young, male patients. This coincides with the characterization of the risk profile of injured, alcoholized patients that can also be found in other studies $[18,19]$.

First, we found no significant differences in the $24 \mathrm{~h}$ mortality. The overall mortality rate showed no significant difference between the groups. Furthermore, the RISC II analysis confirmed our findings (predicted mortality). Looking at all patients in our study collective, no differences in mortality were seen. At most, the length of stay in the hospital was lower in patients with a positive BAL.

We then performed a subgroup analysis to focus on the group of patients with a severe traumatic brain injury (AIS head $\geq 3$ ) to look for further effects that might show different results. However, we showed that the mortality rate in BAL positive patients with TBI was significantly lower than the mortality rate in BAL negative patients with TBI. Additionally, in our subgroup analysis, we observed more patients with an isolated TBI. But the ISS was lower in the BAL positive group. The ISS might have an effect on the higher mortality rate in the BAL negative subgroup.

Age might be influencing the outcome of mortality in this subgroup, too. But we have the same significant age 
Table 4 Outcome according to BAL

\begin{tabular}{|c|c|c|c|c|}
\hline & & BAL negative & BAL positive & $p$-value \\
\hline Mechanical ventilation (days) & Median (IQR) & $0(0-3)$ & $0(0-2)$ & .13 \\
\hline LOS on ICU (days) & Median (IQR) & $3(1-9)$ & $2(1-8)$ & .23 \\
\hline LOS in hospital (days) & Median (IQR) & $13(7-23)$ & $12(6-23)$ & .026 \\
\hline Mortality (24 h) & n (\%) & $266(5.0 \%)$ & $33(4.0 \%)$ & .19 \\
\hline Mortality (in hospital) & n (\%) & $654(12.4 \%)$ & $88(10.6 \%)$ & .14 \\
\hline 95\% Cl for Mortality & & {$[11.5-13.3]$} & {$[8.5-12.7]$} & \\
\hline RISC II Prognose & & $11.7 \%$ & $10.8 \%$ & .97 \\
\hline Subgroup TBI & $n$ & 2092 & 405 & \\
\hline ISS & Mean (SD) & $27(13)$ & $24(12)$ & $<.001$ \\
\hline |solated TB| & n (\%) & $653(31.2 \%)$ & $173(42.7 \%)$ & $<.001$ \\
\hline Mortality (in hospital) & n (\%) & $509(24.3 \%)$ & $68(16.8 \%)$ & .001 \\
\hline $95 \% \mathrm{Cl}$ for mortality & & {$[22.5-26.2]$} & {$[13.1-20.4]$} & \\
\hline RISC II prognosis & Mean & $23.9 \%$ & $18.7 \%$ & $<.001$ \\
\hline
\end{tabular}

LOS, Length of stay. ICU, Intensive Care Unit; TBI, Traumatic brain injury; Cl Confidence Interval

difference when testing for $24 \mathrm{~h}$ mortality and overall mortality in the whole collective. Thus, we do not think that age might be one of the main influencing factors.

Some research groups also found a positive, protective effect of ethanol in patients with moderate and severe TBIs. They showed a significantly lower mortality rate for this group [20].

Tien et al. showed that low to moderate BAL can be beneficial in patients with severe TBI but a high BAL appears to cause an increased risk of mortality during a hospital stay in these patients, which may be related to the adverse hemodynamic and physiological effects in
BAL positive patients [21]. Sperry et al. investigated the influence of alcohol on GCS in TBI patients. They showed that positive BAL in patients with blunt TBI does not lead to clinically significant changes in GCS value [22]. On the other hand, Pandit et al. reported that patients with severe TBI and positive BAL more frequently develop complications during a hospital stay compared to BAL negative patients with TBI. They were therefore unable to detect any neuroprotective effect related to ethanol [23].

In conclusion, a neuro-protective effect of alcohol and the significant survival benefit for alcohol intoxicated

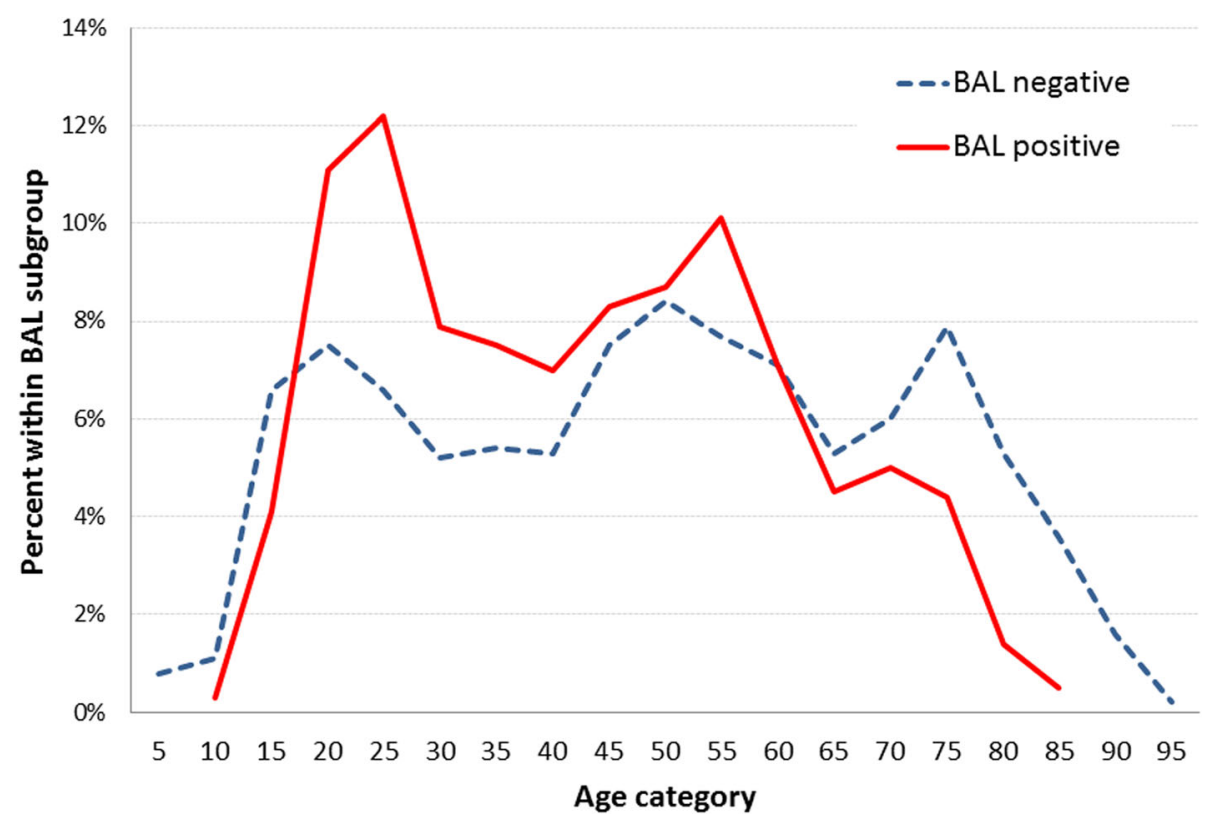

Fig. 1 Age distribution of patients with $(n=832)$ and without $(n=5271)$ positive BAL 
patients with a traumatic brain injury [20,24] cannot be clearly explained and remains of further interest.

In the BAL positive group, a change in physiological parameters such as blood pressure and BE was observed. Alcohol has complex effects on the circulatory system. The relationship between alcohol and high blood pressure is well known, but little is known about the effect of alcohol on the circulation in seriously injured patients. Our results show a small drop in blood pressure in patients in the alcohol group. These results are in line with those of other authors $[5,25]$. In clinical trials, patients with an additional traumatic brain injury had lower amounts of catecholamines in the bloodstream, which in turn resulted in lower systolic blood pressure when admitted to the hospital [26-28].

Patients with a positive BAL have a lower base excess than those in the BAL negative group.

Since alcohol consumption itself leads to lower values of $\mathrm{BE}$ (or higher values of lactate), it is questionable whether these markers could be used as a screening tool for injured intoxicated patients. Normally, the BE is an indicator for tissue hypoperfusion. However, according to a study by Dunne et al., the positive significance of the base excess as a predictive value in relation to mortality remains regarding BAL positive patients [29].

Other studies show a comparable influence on various physiological parameters, in particular on base excess and lactate value, without measuring a difference in mortality [29].

The influence of alcohol on the coagulation system is controversial. In connection with traumatic brain injuries, many studies suggest that with the simultaneous presence of traumatic brain injury and a blood-increased alcohol level, the clotting system is affected. For example, Howard et al. showed that patients with brain injury and positive BAL had a significantly lower incidence of coagulopathies [30]. Since we did not focus on coagulopathies, we cannot make any further reliable statements based on our data but the effect remains of certain interest especially in trauma care.

In our analysis, patients in the alcohol group show an increased risk of falling from a low altitude $(<3 \mathrm{~m})$ compared to patients in the control group. However, we have not been able to prove that patients from the alcohol group are more likely to be involved in accidents with e.g. cars, motorcycles or bicycles. This is likely due to the fact that the majority of BAL positive people no longer drive their own cars, motorbikes or bicycles, but rely on taxis or public transport. The increased number of low falls with an increased rate of severe traumatic brain injuries may be attributed to the fact that the sense of balance under alcohol consumption decreased. Moreover, it can be due to the increased risk of falling with limited protective reflexes.

\section{Limitations}

The analysis presented here as well as the data published in the current literature cannot clarify sufficiently the existing relationship of the effect of ethanol in the context of an existing trauma with and without additional TBI. The discrepancy may be due to the numerous dose-dependent effects of alcohol, the time of exposure in relation to the injury and the nature of the injury and its outcome. In addition, it is very difficult to find comparable collectives in which an effect on hemodynamics and other physiological blood parameters can be investigated. Also, in the present evaluation only correlations, no causal relationships can be described. Additional studies are needed to further investigate the mechanism and possible therapeutic implications of this association.

\section{Conclusion}

This retrospective study of the TraumaRegister DGU analyzes the influence of blood alcohol levels in seriously injured patients. The collective of alcoholic patients shows, among other things, more serious injuries in the area of the head as well as a negative influence on various physiological parameters. However, significant differences in outcome are especially apparent in alcoholic patients with an isolated TBI (AIS head $\geq 3$ ). Comparable collectives need to be found and the effects of alcohol to be further investigated in clinical-experimental studies.

\section{Abbreviation \\ AIS: Abbreviated Injury Scale; BAL: Blood Alcohol Level; BE: Base Excess; Cl: Confidence Interval; DGU: Deutsche Gesellschaft für Unfallchirurgie; GCS: Glasgow Coma Scale; ICU: Intensive Care Unit; INR: International Normalized Ratio; ISS: Injury Severity Score; NISS: New Injury Severity Score; LOS: Length of stay; TBI: Traumatic Brain Injury}

\section{Acknowledgements \\ "Not applicable".}

\section{Authors' contributions}

TB contributed to study design, interpretation of data, recording of paper and analyzing data. RL provided statistical analysis/ advice on study design. $A B, A W, M M, M F$ and $B B$ conceived on the study, provided statistical advice on study design. $\mathrm{AB}, \mathrm{PK}, \mathrm{BB}$ and $\mathrm{MF}$ contributed to analysis and interpretation of data and revision of the article. All authors read and approved the final manuscript for publication.

\section{Funding}

"Not applicable". Open Access funding enabled and organized by Projekt DEAL.

\section{Availability of data and materials}

Please contact author for data requests. Data were provided and analyzed under support of the TraumaRegister DGU ${ }^{\oplus}$ of the German Society of Trauma Surgery (DGU).

\section{Declarations}

Ethical approval and consent to participate "Not applicable". 


\section{Consent for publication}

"Not applicable".

\section{Competing interests}

The corresponding author indicates for himself and his co-authors that there is no conflict of interest.

\begin{abstract}
Author details
'Department of Trauma and Orthopaedic Surgery, University of Witten/ Herdecke, Cologne-Merheim-Medical-Center, Ostmerheimer Street 200, 51109 Cologne, Germany. ${ }^{2}$ Department of Anaesthesiology and Surgical Intensive Care Medicine, University of Witten/Herdecke,

Cologne-Merheim-Medical-Center, Ostmerheimer Street 200, 51109 Cologne, Germany. ${ }^{3}$ Institute for Research in Operative Medicine (IFOM), University of Witten/Herdecke, Ostmerheimer Street 200, 51109 Cologne, Germany. ${ }^{4}$ Working Group of Injury Prevention of the German Trauma Society, The German Trauma Society (DGU), Straße des 17. Juni 106-108, 10623 Berlin, Germany.
\end{abstract}

Received: 26 February 2021 Accepted: 8 July 2021

Published online: 27 July 2021

\section{References}

1. Toroyan T. Global status report on road safety. Inj Prev. 2009;15(4):286. https://doi.org/10.1136/ip.2009.023697.

2. Statistisches Bundesamt. Unfälle unter dem Einfluss von Alkohol oder anderen berauschenden Mitteln im Straßenverkehr 2018. 2019.

3. Bundesamt S. Unfälle von 18- bis 24-Jährigen im Straßenverkehr 2018; 2019.

4. Salim A, Teixeira P, Ley EJ, DuBose J, Inaba K, Margulies DR. Serum ethanol levels: predictor of survival after severe traumatic brain injury. J Trauma. 2009;67(4):697-703. https://doi.org/10.1097/TA.0b013e3181b5dcf2.

5. Scheyerer MJ, Dütschler J, Billeter A, Zimmermann SM, Sprengel K, Werner $\mathrm{CML}$, et al. Effect of elevated serum alcohol level on the outcome of severely injured patients. Emerg Med J. 2013;1(10):813-7. https://doi.org/1 0.1136/emermed-2013-202804.

6. Yaghoubian A, Kaji A, Putnam B, de Virgilio N, de Virgilio C. Elevated blood alcohol level may be protective of trauma patient mortality. Am Surg. 2009; 75(10):950-3.http://www.ncbi.n/m.nih.gov/pubmed/19886142 (accessed 20 Aug2018). https://doi.org/10.1177/000313480907501019.

7. Andersen JA, McLellan BA, Pagliarello G, et al. The relative influence of alcohol and seatbelt usage on severity of injury from motor vehicle crashes. J Trauma. 1990;30(4):415-7.http://www.ncbi.n/m.nih.gov/pubmed/2325170 (accessed 24 Aug2018). https://doi.org/10.1097/00005373-199030040-00007.

8. Porter RS. Alcohol and injury in adolescents. Pediatr Emerg Care. 2000;16(5): 316-20.http://www.ncbi.n/m.nih.gov/pubmed/11063358 (accessed 21 Aug2018). https://doi.org/10.1097/00006565-200010000-00003.

9. Hadjizacharia P, O'Keeffe T, Plurad DS, Green DJ, Brown CVR, Chan LS, et al. Alcohol exposure and outcomes in trauma patients. Eur J Trauma Emerg Surg. 2011;37(2):169-75. https://doi.org/10.1007/s00068-010-0038-5.

10. de Gaetano G, Costanzo S, Di Castelnuovo A, et al. Effects of moderate beer consumption on health and disease: a consensus document. Nutr Metab Cardiovasc Dis. 2016;26(6):443-67. https://doi.org/10.1016/j.numecd.2016.03. 007.

11. Imam I. Alcohol and the central nervous system. Br J Hosp Med. 2010;71: 635-9. https://doi.org/10.12968/hmed.2010.71.11.79653.

12. Majchrowicz E. Metabolic correlates of ethanol, acetaldehyde, acetate and methanol in humans and animals. Adv Exp Med Biol. 1975;56:111-56.http:// www.ncbi.nlm.nih.gov/pubmed/1096549 (accessed 26 Aug2018). https://doi. org/10.1007/978-1-4684-7529-6_5.

13. Ronksley PE, Brien SE, Turner BJ, Mukamal KJ, Ghali WA. Association of alcohol consumption with selected cardiovascular disease outcomes: a systematic review and meta-analysis. Bmj. 2011;342:479. https://doi.org/1 $0.1136 /$ bmj.d671 feb22 1.

14. Borges G, Cherpitel C, Mittleman M. Risk of injury after alcohol consumption: a case-crossover study in the emergency department. Soc Sci Med. 2004;58(6):1191-200.http://www.ncbi.n/m.nih.gov/pubmed/14723913 (accessed 14 Feb2017). https://doi.org/10.1016/S0277-9536(03)00290-9.

15. Ogden $E$, Moskowitz $H$. Effects of alcohol and other drugs on driver performance. Traffic Inj Prev. 2004;5(3):185-98. https://doi.org/10.1080/153 89580490465201.
16. Asmaro K, Fu P, Ding Y. Neuroprotection \&amp; mechanism of ethanol in stroke and traumatic brain injury therapy: new prospects for an ancient drug. Curr Drug Targets. 2013;14(1):74-80.http://www.ncbi.nlm.nih.gov/ pubmed/23170798 (accessed 26 Aug2018). https://doi.org/10.2174/13894 5013804806505

17. Opreanu RC, Kuhn D, Basson MD. Influence of alcohol on mortality in traumatic brain injury. J Am Coll Surg. 2010;210(6):997-1007. https://doi. org/10.1016/j.jamcollsurg.2010.01.036.

18. Cornwell EE, Belzberg H, Velmahos $\mathrm{G}$, et al. The prevalence and effect of alcohol and drug abuse on cohort-matched critically injured patients. Am Surg. 1998;64(5):461-5.http://www.ncbi.n/m.nih.gov/pubmed/9585786 (accessed 21 Aug2018).

19. Sloan EP, Zalenski RJ, Smith RF, et al. Toxicology screening in urban trauma patients: drug prevalence and its relationship to trauma severity and management. J Trauma. 1989;29(12):1647-53.http://www.ncbi.nlm.nih.gov/ pubmed/2593195 (accessed 28 Aug2018). https://doi.org/10.1097/000053 73-198912000-00011.

20. Berry C, Salim A, Alban R, Mirocha J, Margulies DR, Ley EJ. Serum ethanol levels in patients with moderate to severe traumatic brain injury influence outcomes: a surprising finding. Am Surg. 2010;76(10):1067-70.http://www. ncbi.nlm.nih.gov/pubmed/21105611 (accessed 21 Aug2018). https://doi. org/10.1177/000313481007601009.

21. Tien HCN, Tremblay LN, Rizoli SB, Gelberg J, Chughtai T, Tikuisis P, et al. Association between alcohol and mortality in patients with severe traumatic head injury. Arch Surg. 2006;141(12):1185-91; discussion 1192. https://doi. org/10.1001/archsurg.141.12.1185.

22. Sperry JL, Gentilello LM, Minei JP, Diaz-Arrastia RR, Friese RS, Shafi S. Waiting for the patient to 'sober up': effect of alcohol intoxication on Glasgow coma scale score of brain injured patients. J Trauma. 2006;61(6):1305-11. https:// doi.org/10.1097/01.ta.0000240113.13552.96.

23. Pandit V, Patel N, Rhee P, Kulvatunyou N, Aziz H, Green DJ, et al. Effect of alcohol in traumatic brain injury: is it really protective? J Surg Res. 2014; 190(2):634-9. https://doi.org/10.1016/j.jss.2014.04.039.

24. Talving P, Plurad D, Barmparas G, DuBose J, Inaba K, Lam L, et al. Isolated severe traumatic brain injuries: Association of Blood Alcohol Levels with the severity of injuries and outcomes. J Trauma Inj Infect Crit Care. 2010;68(2): 357-62. https://doi.org/10.1097/TA.0b013e3181bb80bf.

25. Molina PE, Zambell KL, Norenberg K, Eason J, Phelan H, Zhang P, et al. Consequences of alcohol-induced early dysregulation of responses to trauma/hemorrhage. Alcohol. 2004;33(3):217-27. https://doi.org/10.1016/j.a Icohol.2004.07.002.

26. Nylén K, Ost M, Csajbok LZ, et al. Increased serum-GFAP in patients with severe traumatic brain injury is related to outcome. J Neurol Sci. 2006;240(12):85-91. https://doi.org/10.1016/j.jns.2005.09.007.

27. Türeci E, Dashti R, Tanriverdi T, Sanus GZ, Öz B, Uzan M. Acute ethanol intoxication in a model of traumatic brain injury: the protective role of moderate doses demonstrated by immunoreactivity of synaptophysin in hippocampal neurons. Neurol Res. 2004;26(1):108-12. https://doi.org/10.11 79/016164104773026633

28. Woolf PD, Cox C, Kelly M, McDonald JV, Hamill RW Alcohol intoxication blunts sympatho-adrenal activation following brain injury. Alcohol Clin Exp Res 1990;14:205-9. http://www.ncbi.n/m.nih.gov/pubmed/2190486. Accessed 27 Mar 2019, 2. https://doi.org/10.1111/j.1530-0277.1990.tb00473.x.

29. Dunne JR, Tracy JK, Scalea TM, Napolitano LM. Lactate and base deficit in trauma: does alcohol or drug use impair their predictive accuracy? J Trauma. 2005;58(5):959-66.http://www.ncbi.nlm.nih.gov/pubmed/15920409 (accessed 21 Aug2018). https://doi.org/10.1097/01.TA.0000158508.84009.49.

30. Howard BM, Kornblith LZ, Redick BJ, Vilardi RF, Balhotra KS, Crane JM, et al. The effects of alcohol on coagulation in trauma patients. J Trauma Acute Care Surg. 2014;77(6):865-72. https://doi.org/10.1097/TA.0000000000000357.

\section{Publisher's Note}

Springer Nature remains neutral with regard to jurisdictional claims in published maps and institutional affiliations. 